\title{
Ellipsis as obliteration: Evidence from Bengali negative allomorphy
}

\author{
Neil Banerjee*
}

\begin{abstract}
Bengali has two negative markers: $n i$ with perfects, and $n a$ everywhere else. When a perfect is elided, however, only the elsewhere form is permissible. Hence, in Bengali, ellipsis bleeds allomorphy. Ellipsis in Bengali is analysed as PF deletion, since differential object marking and quirky case are preserved out of ellipsis sites. Given these facts, this paper argues that in a Distributed Morphology framework, ellipsis in Bengali is implemented as terminal obliteration prior to Vocabulary Insertion. This contrasts with Irish, where it appears stress placement bleeds ellipsis. Some implications for the timing of ellipsis cross-linguistically are discussed.
\end{abstract}

Keywords. allomorphy; ellipsis; timing; Bengali; Irish

1. Introduction. Bengali (Eastern Indo-Aryan, also called Bangla) has two morphemes that negate non-copular clauses. The more common negation, $n a$, is used to negate non-perfect clauses by being added clause-finally as shown in (1) and (2).
a. behala baja-i
violin play-1
'I play the violin.'

b. behala baja-i na

violin play-1 NA

'I don't play the violin.'

(2)
a. am-ța khe-lam
mango-CL eat-1.PST
'I ate the mango.'
b. am-ta khe-lam na
mango-CL eat-1.PST NA
'I did not eat the mango.'

Perfect verbs, however, behave differently. The affirmative perfect, shown in (3-a) cannot be negated by appending clause-final $n a$, as shown in (3-b). Instead, they must be negated with the special negation $n i$ as in (3-c), along with some concomitant changes to the verbal morphology. The affirmative perfect as in (3-a) requires the perfect marker - $e$ and the auxiliary $c h(i)$-, however when negated with $n i$ as in (3-c), both of these are missing.
a. ei
boi-ța por-e ch-i
DEM.PROX book-CL read-PRF AUX-1
'I have read this book.'
b. *ei boi-ța por-e ch-i na
DEM.PROX book-CL read-PRF AUX-1 NA

*Thanks to Adam Albright, Jonathan Bobaljik, Christos Christopoulos, Sabine Iatridou, Filipe Kobayashi, and Stanislao Zomp for discussion and comments, Dmitry Privoznov for inspiration, Tanya Bondarenko, Colin Davis, and David Pesetsky for suggestions, Debal Banerjee, Sagarika Banerjee, and Shoma Bhattacharya for judgements, and to the audience at LSA 2020, particularly for comments. This research was supported by the Social Sciences and Humanities Research Council of Canada Doctoral Fellowship 752-2017-0069. Author: Neil Banerjee, Massachusetts Institute of Technology (neilb@ mit.edu). 


\section{Int: 'I have not read this book.' \\ c. ei boi-ta por-i ni \\ DEM.PROX book-CL read-1 NI \\ 'I have not read this book.'}

This paper investigates the distribution of negative forms in Bengali and argues that it is best analysed as the result of contextual allomorphy, contra the semantic proposal put forth by Ramchand (2004). Despite $n a$ being impossible with perfect verbs as shown in (3), if a perfect verb undergoes ellipsis, the negation which survives is obligatorily $n a$. Since the "correct" negation is unavailable with an elided perfect, ellipsis appears to bleed the choice of negative morpheme in Bengali. In it I argue that given this bleeding relation between ellipsis and negation choice, ellipsis in a Distributed Morphology framework (Halle \& Marantz 1993, 1994) must be modelled as terminal node obliteration (Arregi \& Nevins 2007).

The rest of the paper is structured as follows. Section 2 discusses properties of Bengali negation and the structure of the clause. Section 3 presents the distribution of negative forms and argues on the basis of ellipsis facts that a semantic approach to the distribution is unsuccessful. Section 4 presents a morphological account couched in a Distributed Morphology framework and discusses issues of locality and directionality of allomorphy. Section 5 investigates properties of gapping in Bengali and argues that given its interaction with negative allomorphy, it should be modelled as terminal node obliteration. Section 6 compares the Bengali data to Irish, where the realisation of verum focus bleeds ellipsis, and discusses cross-linguistic implications. Section 7 concludes.

2. Properties of Bengali negation and the clause. Before understanding how negation interacts with the perfect in Bengali, we must first understand some of the properties of negation itself. Firstly, it appears that negation in Bengali is a head, not a phrase. This is evidenced by the 'Why not?' test from Zeijlstra (2008). Since 'why' is a phrasal adjunct, if the exponent of negation in a language is phrasal, it is predicted to be usable to form the question 'why not?'. As shown in (4-a), neither $n a$ nor $n i$ in Bengali is capable of hosting the phrasal adjunct 'why'. Furthermore, despite allowing a large degree of freedom in scrambling, Bengali does not permit any phrasal material to intervene between the verb and negation, as shown in (4-b).
a. *kèno na/ni?
why NA/NI
'Int: Why not?'
b. $\quad\{$ mach $\}$ kha-i $\{*$ mach $\}$ na/ni $\{$ mach $\}$
$\{$ fish $\}$ eat $1\{*$ fish $\}$ NA/NI $\{$ fish $\}$
'I $\{$ don't eat/haven't eaten $\}$ fish.'

Both of these facts strongly suggest that negation in Bengali is always a head. Turning now to its position in the spine, we see that both negations scope over subjects.
a. keu dek-e ch-e
somebody call-prf AUX-3
'Somebody has called.'
b. keu dak-e na/ni
somebody call-3 NA/NI
'Nobody calls/has called.' 
The subject in in both (5-a) and (5-b) is existential, as can be seen from its meaning in (5-a). In (5-b), we see that this existential subject is interpreted under the scope of negation. Following Zeijlstra (2008), if negation only has surface scope, it must be licensing the subject from above Spec-Infl. This suggests a high position for negation. This is supported by evidence from morpheme ordering. As per the Mirror Principle Baker (1985), syntactic hierarchy relationships are reflected in the ordering of morphemes on the surface. If morpheme A is structurally closer to a root than morpheme $\mathrm{B}$, the exponent of $\mathrm{A}$ must be at least as close to the exponent of the root as the exponent of B. This lets us 'read' some amount of hierarchical information from the order of morphemes. For example, given the structure in (6-a), the possible surface forms are given in (6-b).

$$
\begin{array}{ll}
\text { a. } & \\
\text { b. } & \\
& \text { (i) } \text { V-A-B } \\
& \text { (ii) } \mathrm{B}-\mathrm{V}-\mathrm{A} \\
\text { (iii) } \mathrm{A}-\mathrm{V}-\mathrm{B} \\
\text { (iv) } \mathrm{B}-\mathrm{A}-\mathrm{V} \\
\text { (v) } * \mathrm{~A}-\mathrm{B}-\mathrm{V} \\
\text { (vi) } * \mathrm{~V}-\mathrm{B}-\mathrm{A}
\end{array}
$$

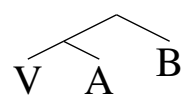

In the case of Bengali, we saw in (1) and (2) that the exponent of negation is always further from the verbal root than the exponent of Infl. Furthermore, we saw from (3-a) that the exponent of the perfect is adjacent to the verb root. Since all of the morphemes in question are suffixes there is no ambiguity about the structural relations between them (unlike (6-b-ii) and (6-b-iii) which could both be derived from structures where B c-commands A or vice versa). We can conclude from this that the Bengali verbal spine looks as shown in (7).

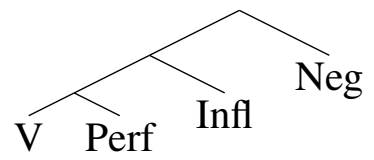

Recall that affirmative perfects involve not only an overt exponent of Perf, but also an obligatory auxiliary that carries inflectional morphology as in (3-a). I assume that the auxiliary, which is absent in the negative, is inserted in Infl to carry inflectional morphology which cannot be hosted by the verb. See Bjorkman (2011) for details of this general approach to overflow morphology. In brief, the idea is that the verb's inflectional morphology slot is occupied by the exponent of the perfect, and thus an auxiliary must be inserted post-syntactically into Infl to carry the tense and agreement morphology to prevent it from being stranded without a host.

With this information in place, we can now turn to understanding the distribution of the two negative forms.

3. The distribution of $\boldsymbol{n a}$ and $\boldsymbol{n i}$. The two negative forms in Bengali appear to be in complementary distribution: $n i$ appears only with perfects and nowhere else, while $n a$ appears everywhere except with perfects. This leads Ramchand (2004) to propose that they in fact have different semantics, which in turn accounts for why they cannot occur in the same environments. For her, $n a$ is a vP-level negative quantifier over events, while $n i$ is an Infl-level negative quantifer over times. She does not provide lexical entries for the quantifiers, but describes them $n a$ 
as meaning "no event of the specified type occurred at that moment", while $n i$ "states that for no time at all (in the discourse context) did an event of the specified type occur". Adopting the view from Parsons (1990) that all events have resultant states, and that perfects describe specifically such a state straightforwardly predicts the unavailability of $n a$ with perfects. This is because a perfect would introduce an event and its resultant state, but $n a$ would assert that the resultant state did not exist. This is incoherent in the system, as all events introduce resultant states.

However, we have already seen reasons to be concerned about this approach. Firstly, $n a$ and $n i$ both appear to be at the same height, which Ramchand (2004) does not predict. Furthermore, we see below that despite na being unavailable with overt perfects, it is surprisingly obligatory with elided perfects.

$$
\begin{aligned}
& \text { ilis ònek bar khe-e ch-i, (kintu) magur kòkkhono } \Delta \text { na/*ni } \\
& \text { hilsa many times eat-PRF AUX-1, (but) catfish ever } \Delta \text { NEG } \\
& \text { 'I have eaten hilsa many times, but not ever catfish.' }
\end{aligned}
$$

In (8), $\Delta$ marks the ellipsis site. This is an example of gapping, where TP ellipsis occurs, but some constituents survive (namely the adverb ever and the noun catfish). Note that since negation is present, but no inflectional material survives, the ellipsis site must minimally contain Infl. If the ellipsis site were smaller, we might expect to see an auxilary to carry inlfection. The absence of such an auxiliary indicates that the ellipsis site contains Infl and everything below it. If $n a$ were in fact a vP-level negative quantifier, it should be within the ellipsis site, and thus gapping of the sort seen above should never leave $n a$ as the surviving negation, only $n i$ which for Ramchand (2004) is higher than Infl. Furthermore, we see that contra the predictions of Ramchand (2004), the presence of $n a$ with a perfect does not cause a contradiction.

One might worry at first that the above construction may not involve clausal negation, but rather constituent negation of one of the surviving constituents (perhaps the adverb). However, Bengali does not appear to have constituent negation at all. Instead, clausal negation is used with an existentially quantifier DP, as shown for subjects in (9) below.
a. *aj cithi na/ni e-lo today letter NEG arrive-3.PST Int: 'No letters arrived today.'
b. aj kono cithi e-lo na today some letter arrive-3.PST NEG 'No letters arrived today.'

Given this, we can safely conclude that (8) does indeed involve clausal negation, and so the absence of $n i$ with an elided perfect is indeed problematic for the semantic account of the distribution of negative forms in Bengali. Thus, $n a$ and $n i$ are best not analysed as distinct negative quantifiers at different heights, but rather by some other means.

Consider the examples below, illustrating a minimal pair of a negative habitual and a negative perfect.

$$
\begin{aligned}
& \text { a. ol kha-i na } \\
& \text { jackfruit eat-1 NA } \\
& \text { 'I do not eat jackfruit.' }
\end{aligned}
$$



b. ol kha-i ni
jackfruit eat-1 NI
'I have not eaten jackfruit.'

Since the only difference is in the form of negation, it is reasonable to take as a starting point, the idea that $n i$ contains information both about negation and the perfect: it is a portmanteau.

4. Building portmanteaux in Distributed Morphology. Realizational theories of morphology in the tradition of Distributed Morphology (Halle \& Marantz 1993, 1994) and related traditions have proposed a variety of mechanism to derive portmanteaux. Note that I use portmanteau as a pretheoretic term, referring to a single exponent realising multiple features, as appears to be the case with Bengali $n i$ realising both negation and the perfect.

Oldest among the analyses for portmanteau was Halle \& Marantz (1993) themselves, who proposed that they could be formed through the processes of head movement followed by fusion. Head movement brings two adjacent heads into a sisterhood relationship, and then fusion replaces the two sister terminals with a single terminal consisting of the union of their features. Insertion then targets this fused terminal, resulting in the inserted vocabulary item exponing the features of both heads. However, as observed by Travis (1984), head movement is subject to locality conditions, which she termed the Head Movement Constraint. This prohibits head movement from skipping intervening heads, meaning this is not a viable option for accounting for Bengali. Recall that the structure of the spine put Infl as intervening between Perf and Neg. If head movement were to occur, there would be no way for Perf to skip over Infl and merge with Neg. Yet in the realisation of negative perfects as in (11), we see that agreement is exponed separately from $n i$, and hence Infl has clearly not fused with Perf and Neg.
am kha-i ni
mango eat-1 neg.perf
'I have not eaten (the) mango.'

Svenonius (2016) and Ostrove (2018) propose that portmanteaux can be formed from spans and stretches respectively. Spans are sequences of structurally adjacent heads, while stretched are sequences of linearly adjacent feature bundles. Crucially, since both of these options require adjacency, the non-local nature of the relationship between Perf and Neg mean that neither spans nor stretches can straightforwardly account for why $n i$ expones both Neg and Perf.

Trommer (1999) however, proposes that portmanteaux may be formed as the result of a conspiracy of two separate cases of contextual allomorphy. One of the two terminals that the portmanteau realises is exponed as the apparent portmanteau morpheme, while the other is realised as null. This approach is not inherently restricted to adjacency like previous approaches, and so can be used to model the case of Bengali. I adopt the common view in Distributed Morphology that vocabulary insertion proceeds root-outwards and that the Subset Principle Halle \& Marantz (1993) governs insertion. The vocabulary insertion rules below are able to account for the distribution of negative morphemes as well as the overt realisation of the perfect.

$$
\begin{array}{ll}
\text { a. } & \text { Perf } \left.\left.\rightarrow \varnothing / /_{--}\right] \ldots\right] \mathrm{Neg} \\
\text { b. } & \text { Perf } \rightarrow-\mathrm{e}^{1}
\end{array}
$$

\footnotetext{
${ }^{1}$ The issue of vowel height in verb roots is not discussed in this paper. The perfect triggers raising of root vowels
} 
c. $\quad$ Neg $\rightarrow$ ni / Perf ] ...] --

d. $\quad$ Neg $\rightarrow$ na

In the particular case of interest, a negative perfect, the structure accessible to morphology will be the structure given in (7), where both Perf and Neg are present. This is repeated below for readability.

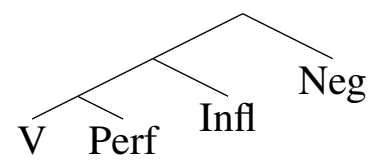

After inserting the vocabulary item for the verb, the rule in (12-a) will find its contextual specification met, and thus Perf will be realised as null. Infl will be inserted and subsequently rule (12-c) will apply to insert $n i$ as the realisation for Neg in the context of a (silent) perfect.

The vocabulary insertion rules posited above raise questions about the restrictions of locality and directionality in contextual allomorphy. Bobaljik (2000) has proposed that vocabulary insertion proceeds root outwards and is replacive, in that abstract features on terminals are replaced by their phonological exponents at insertion. This predicts that inward-sensitive allomorphy should only be phonologically conditioned, as morphosyntactic features will not be present below the site of insertion. Yet the rule in (12-c) crucially requires inward sensitivity to the morphosyntactic feature of the Perf head. This is because the phonological form of the verb root and agreement is identical in the negative perfect and the negative habitual, as shown in (10), and repeated below.
a. ol kha-i na
jackfruit eat-1 NEG
'I do not eat jackfruit.'
b. ol kha-i ni
jackfruit eat-1 NEG.PRF
'I have not eaten jackfruit.'

If the insertion algorithm only had access to the phonological content below Neg, it would never be able to distinguish the negative perfect from the negative habitual, and thus not know whether to insert $n i$ or $n a$. So, contra Bobaljik (2000), inward-sensitivity to morphosyntactic features appears to be required. This is in line with proposals in Carstairs (1987) and CarstairsMcCarthy (2001), which maintain that a certain degree of inward-sensitivity must be available to account for inflectional patterns in Latin for example. Since inward sensitivity in Bobaljik's system was achieved through a replacive insertion algorithm, I will adopt a system that is additive, not replacive. Insertion will still proceed root-outward, however inserting an exponent into a terminal will not replace the abstract features.

Locality restrictions on contextual allomorphy have also been investigated in the literature, leading to claims that they require either structural adjacency (Embick 2010) or string adjacency (Arregi \& Nevins 2012). These analyses, however, focussed on root allomorphy conditioned by affixes. Moskal (2015) observes on the basis of a survey of 193 languages (Veseli-

one step systematically. This is a regular morphophonological rule triggered by the overt exponent of the perfect. The alternation is sometimes obscured however, because high vowels in agreement suffixes triggers partial height harmony in root vowels as well. See Sanyal (2012) for a discussion of the Bengali vowel system in verbal paradigms. 
nova 2006), that the locality restrictions only seem to apply to lexical material. She analyses this restriction as the result of a phase boundary introduced by the category label, predicting that affixal allomorphy conditioned by other affixes should be permissable at longer distances so long as no category labels intervene. Category labels have been systematically disregarded in this paper because they commit to a particular notion of roots as acategorial - a position that is not required for any of the claims in this paper. So it is sufficient to note that the adjacency requirements on allomorphy appear to be robustly instantiated only for root allomorphy, and that proposals exist which predict affixal allomorphy to be unbounded. Since the analysis proposed here involves conditioning Neg and Perf on each other, both participants in the allomorphic processes are affixes, and so no locality effects should be expected in a view like that of Moskal (2015).

5. Ellipsis and allomorphy. Before considering the interaction of ellipsis and allomorphy in Bengali, it is worth establishing the nature of the gapping examples at hand. Proposals to analyse the elided material range from silent pronouns without internal structure (i.e. deep anaphors in Hankamer \& Sag (1976)) to full-fledged structures that are interpreted but not pronounces (i.e. surface anaphors in Hankamer \& Sag (1976)). Bengali gapping appears to fall into the second category, since differential object marking (DOM) and quirky case assignment still apply to the ungapped elements.

Bengali DOM requires oblique case marking on all human non-subjects in all clausetypes. If a clause undergoes gapping and a human non-subject survives, it must also carry oblique case, as shown in (15).

$$
\begin{aligned}
& \text { Ruma-ke cin-i, Ram-*(ke) } \Delta \text { na } \\
& \text { Ruma-OBL know-1, Ram-*(OBL) } \Delta \text { NEG } \\
& \text { 'I know Ruma, not Ram.' }
\end{aligned}
$$

Quirky genitive case is assigned in Bengali to the subjects of certain experiencer verbs, like 'feel'. If such a verb is gapped and the subject is stranded, it must still show the quirky genitive case, as shown in (16).

$$
\begin{aligned}
& \text { Asa-r am bhalo lag-e, Ram-*(er) } \Delta \text { na } \\
& \text { Asa-GEN mango good feel-3, Ram-*(gen) } \Delta \text { NEG } \\
& \text { 'Asa likes mangoes, Ram does not.' }
\end{aligned}
$$

Since these processes require a certain configuration between a DP and a verb and can apply across an ellipsis boundary, it demonstrates that the structure of the verbal domain must be present within the ellipsis site in order to trigger the process on the unelided DP. Thus, gapping in Bengali is the result of deletion of structure, not semantic copying. I assume a featural account of ellipsis licensing, following Merchant (2001). In this view, constituents meant for ellipsis are marked with a feature (denoted by Merchant as E). At LF, the identity conditions for ellipsis are checked for whatever constituents bear this feature, while at PF, constituents marked with this feature are somehow silenced. The question then is how are constituents marked with these features silenced? For simplicity, I assume that the E-feature percolates down to the terminals dominated by the maximal node marked by E. This allows us to consider only operations that act on terminals. 
There are, at first glance, three possible options to silence them.

(17) a. Phonology penalises correspondence with an E-marked terminal.

b. Morphology inserts $\varnothing$ into E-marked terminals.

c. Morphology obliterates the contents of all E-marked terminals prior to insertion.

Given that the model of morphology assumed does not output multiple candidate structures, the option in (17-a) must be discounted. If ellipsis only applied in the phonology, Neg would be exponed by $n i$ in the case of a perfect well before ellipsis occurred, and thus ellipsis would not be able to bleed allomorphy. Recall that the insertion rule for $n i$ relied on inward-sensitivity to morphosyntactic features, and that insertion was argued to be non-replacive. This predicts that the option in (17-b) would also insert $n i$ into Neg. Consider the point of insertion of Neg in a system where ellipsis is implemented as option (17-b).

Ellipsis as $\varnothing$ insertion

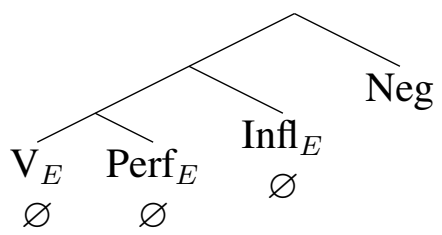

Since the terminal Perf is still present, insertion rule (12-c), which was conditioned on the presence of a silent Perf, would apply and insert $n i$ as the exponent of Neg contra the observed facts.

Hence we are left with the option in (17-c): ellipsis is obliteration. Obliteration, proposed by Arregi \& Nevins (2007), is a form of impoverishment. Impoverishment generally is the deletion of some features of a terminal in a certain configuration. Obliteration is the name given to maximal impoverishment, where all features of a terminal are deleted. Obliterated terminals are not able to condition allomorphy because they have no featural content. Thus, ellipsis in Bengali is able to bleed negative allomorphy.

6. Comparison with Irish. The conclusion from the investigation into Bengali negation and ellipsis was that TP ellipsis in Bengali was implemented as obliteration prior to vocabulary insertion. Naturally, we would want to know whether TP ellipsis is always obliteration. Irish seems to indicate that the answer is 'no'. Verum focus and TP ellipsis interact in Irish in nearly the opposite way as in Bengali, namely, ellipsis is bled by stress placement for focus. On its own, focus bleeding ellipsis may not be surprising since focussed elements are precisely those which escape gapping. However in Irish, verum focus results in a PF-LF mismatch. While verum focus is often analysed as focus on the polarity of the clause, in Irish it is realised as stress on a pronominal subject.

$$
\begin{aligned}
& \mathrm{N} \quad \text { rachaidh SÉ síos! } \\
& \text { NEG.FIN go.FUT 3M down! } \\
& \text { 'It won't GO down!' }
\end{aligned}
$$

(Bennett et al. 2019; 17)

The form of ellipsis that this interacts with is called responsive ellipsis, which is the way Yes/No questions are answered in Irish. Lacking words for "yes" and "no", Irish repeats the verb in the question with the appropriate polarity. Bennett et al. (2019) assume this involves the verb moving to the polarity head, followed by TP ellipsis. Crucially, as shown in (20) below, the 
subject pronoun can normally not survive in responsive ellipsis.

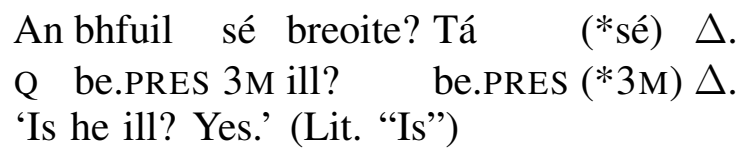

(Bennett et al. 2019; 29)

When both verum focus and responsive ellipsis happen together, however, the subject pronoun bearing stress is allowed to survive.

$$
\begin{aligned}
& \text { An raibh sí ag scríobh litreacha? Bhí SÍ } \Delta \text { ! } \\
& \text { Q be.PST 3F PREP write letters? be.PST 3F } \Delta \text { ! } \\
& \text { 'Was she writing letters? YES!' (Lit. "Was she!") }
\end{aligned}
$$

(Bennett et al. 2019; 29)

The surviving subject pronoun is crucially not the focalised item, yet it is able to survive ellipsis when it bears stress for verum focus. Since stress placement can bleed ellipsis in Irish, Bennett et al. (2019) analyse ellipsis as begin implemented in the phonology proper. Both ellipsis and stress assignment are violable constraints. In Irish stress assignment constraints outrank ellipsis-enforcing constraints, hence verum stress assignment proceeds normally at the cost of pronouncing elided material.

This is crucially different to the mechanism proposed for Bengali, where TP ellipsis involves terminal node obliteration in the earliest part of morphology. Given the radically differing natures of TP ellipsis that Bengali and Irish seem to point to, two possible stances are possible.

On one hand, it is possible that the analyses are both correct as they stand, and that ellipsis, even of constituents of the same size, is not a cross-linguistically uniform process. Rather, than being an operation, ellipsis is better thought of as a description of the result of different processes cross-linguistically aiming to achieve the same goal of silencing material deemed somehow unnecessary. In this view, ellipsis is emergent behaviour.

On the other hand, it is possible that TP ellipsis is in fact cross-linguistically a single process. The different analyses seemingly required for Bengali and Irish would then indicate that the frameworks assumed for morphology and/or stress placement are not optimal. If the Bengali and Irish cases are to be unified, morphology and prosody must happen in the same module. Recent work has argued for such conclusions, although in two different directions. Richards (2016), for example, argues that prosodic information is present in the syntax itself, and syntactic movement can be to satisfy prosodic requirements. If this view is adopted, perhaps the mechanism for realising verum focus on the subject pronoun in Irish involves moving the pronoun out of the ellipsis site in the narrow syntax itself. Ellipsis could then proceed as obliteration without accidentally deleting the subject just in case it has undergone movement. Conversely, it is possible that morphology and phonology occur within the same Optimality Theoretic (Prince \& Smolensky 2004) module, as has been argued by Wolf (2008). In this view, insertion is governed by a series of violable constraints which are able to make reference to the correspondence between terminal features and their exponents. Bengali then would involve the ellipsis enforcing constraints crucially outranking the allomorphy governing ones. The deletion of the ellipsis site would then need to eliminate any benefit from inserting anything but the default allomorph of negation. Exploring all of these possibilities in more detail is left for future work. 
7. Conclusion. This paper has investigated the distribution of negative forms in Bengali clausal negation. It was shown, using data from gapping constructions, that the alternation is best analysed as allomorphic, not semantic. Given that one allomorph of negation, $n i$, was analysed as a contextual allomorph in the presence of a silent Perf head, a model of morphology that permits inward-sensitivity to morphosyntactic features was adopted. Since ellipsis, which was shown to be PF deletion, is able to bleed this choice of negation, it was argued that TP ellipsis must be implemented as termial node obliteration in Bengali. However, comparison with Irish showed that this model of TP ellipsis may not be universally applicable. Given that Irish seems to demonstrate stress placement bleeding ellipsis, reconciling Bengali and Irish requires the adoption of one of two stances. The first option is that ellipsis is emergent behaviour with cross-linguistically variable processes and timings. The second option is that our understanding of PF needs to be rethought. Either prosodic information is present in the narrow syntax and can influence it, or allomorphy occurs in the same module as focus stress placement. Investigating the implications of each of these stances is left for future work.

\section{References}

Arregi, Karlos \& Andrew Nevins. 2007. Obliteration vs. impoverishment in the Basque g-/zconstraint. In University of Pennsylvania Working Papers in Linguistics, vol. 13.1.

Arregi, Karlos \& Andrew Nevins. 2012. Morphotactics. Dordrecht: Springer. http://dx.doi.org/10.1007/978-94-007-3889-8.

Baker, Mark. 1985. The mirror principle and morphosyntactic explanation. Linguistic Inquiry 16(3). 373-415.

Bennett, Ryan, Emily Elfner \& James McCloskey. 2019. Prosody, focus, and ellipsis in Irish. Language 95(1). 66-106. http://dx.doi.org/10.1353/lan.2019.0012.

Bjorkman, Bronwyn. 2011. BE-ing default: The morphosyntax of auxiliaries. Massachusetts Institute of Technology doctoral dissertation.

Bobaljik, Jonathan David. 2000. Implications of Itelmen Agreement Asymmetries. In Proceedings of the 25th Annual Meeting of the Berkeley Linguistics Society. 300-310.

Carstairs, Andrew. 1987. Allomorphy in inflexion. London: Croom Helm.

Carstairs-McCarthy, Andrew. 2001. Grammatically conditioned allomorphy, paradigmatic structure, and the Ancestry Constraint. Transactions of the Philological Society 99(2). 223245. http://dx.doi.org/10.1111/1467-968X.00081.

Embick, David. 2010. Localism versus Globalism in Morphology and Phonology. Cambridge, MA: The MIT Press. http://dx.doi.org/10.7551/mitpress/9780262014229.001.0001.

Halle, Morris \& Alec Marantz. 1993. Distributed Morphology and the pieces of inflection. In Kenneth Hale \& Jay Keyser (eds.), The View from Building 20, 111-176. Cambridge, US: MIT Press.

Halle, Morris \& Alec Marantz. 1994. Some key features of Distributed Morphology. In Andrew Carnie \& Heidi Harley (eds.), Papers on Phonology and Morphology, vol. 21, 275288. Cambridge, US: MIT Working Papers in Liguistics.

Hankamer, Jorge \& Ivan Sag. 1976. Deep and surface anaphora. Linguistic Inquiry 7. 391-428.

Merchant, Jason. 2001. The syntax of silence: Sluicing, islands, and the theory of ellipsis (Oxford Studies in Theoretical Linguistics 1). Oxford; New York: Oxford University Press.

Moskal, Beata. 2015. Domains on the border: Between morphology and phonology. University of Connecticut at Storrs doctoral dissertation. 
Ostrove, Jason. 2018. Stretching, spanning, and linear adjacency in Vocabulary Insertion. Natural Language \& Linguistic Theory 36(4). 1263-1289. http://dx.doi.org/10.1007/s11049-0189399-y.

Parsons, Terence. 1990. Events in the semantics of English. Cambridge, MA: MIT Press.

Prince, Alan \& Paul Smolensky (eds.). 2004. Optimality theory. Oxford: Blackwell. http://dx.doi.org/10.1002/9780470759400.

Ramchand, Gillian. 2004. Two Types of Negation in Bengali. In Marcel den Dikken, Liliane Haegeman, Joan Maling, Veneeta Dayal \& Anoop Mahajan (eds.), Clause structure in South Asian languages, vol. 61. 39-66. Dordrecht: Springer Netherlands. http://dx.doi.org/10.1007/978-1-4020-2719-2_2.

Richards, Norvin. 2016. Contiguity theory (Linguistic Inquiry Monographs 73). Cambridge, Massachusetts: The MIT Press.

Sanyal, Paroma. 2012. Phonology to morphophonology: Reanalyzing Bangla verbs. English and Foreign Languages Journal 3(2). 65-84.

Svenonius, Peter. 2016. Spans and words. In Heidi Harley \& Daniel Siddiqi (eds.), Morphological metatheory. 199-220. Amsterdam: John Benjamins.

Travis, Lisa. 1984. Parameters and effects of word order variation. Cambridge, MA: Massachusetts Institute of Technology dissertation.

Trommer, Jochen. 1999. Morphology consuming syntax’ resources. Ms., Universität Potsdam.

Veselinova, Ljuba N. 2006. Suppletion in verb paradigms: Bits and pieces of the puzzle, vol. 67 Typological Studies in Language. Amsterdam: John Benjamins Publishing Company. http://dx.doi.org/10.1075/tsl.67.

Wolf, Matthew. 2008. Optimal interleaving: Serial phonology-morphology interaction in a constraint-based model. Amherst, US: University of Massachusetts, Amherst doctoral dissertation.

Zeijlstra, Hedde. 2008. Negative concord is syntactic agreement. Lingbuzz/000645. 